\title{
Assessment of Molecular Diversity in Castor Genotypes Using SSR Markers
}

\author{
Harshita J. Kachhadiya $^{1 *}$, R.B. Madaria ${ }^{2}$, Rushita V. Bhadani ${ }^{1}$, \\ Abhijeeta K. Nandha ${ }^{1}$, Nidhi Savaliya ${ }^{1}$ and Virali Antala ${ }^{1}$ \\ ${ }^{1}$ Department of Biotechnology, Junagadh Agricultural University, \\ Junagadh-362001, Gujarat, India \\ ${ }^{2}$ Main Oilseeds Research Station, Junagadh Agricultural University, \\ Junagadh-362001, Gujarat, India \\ *Corresponding author
}

\section{A B S T R A C T}

Castor (Ricinus communis L.) is a plant that is commercially very important to the world. It is produced in about 30 countries lying in the tropical belt of the world. It is an important plant for production of industrial oil. Assessment of

Keywords

Castor (Ricinus communis L.), Euphorbiaceae

Article Info

Accepted: 07 June 2019 Available Online: 10 July 2019 genetic diversity of a crop species is a prerequisite to its improvement; hence it is important to identify the genetic diversity of castor genetic resources for development of improved cultivars. The aim of the present study was to study the molecular diversity for varietal identification and phylogenetic relationships among twenty castor genotypes and identify those with distinct DNA profiles. Thirty SSRs primers were used, out of which 25 polymorphic primers revealed $53.33 \%$ polymorphism among the castor genotypes. Dendrogram was constructed using UPGMA method which revealed distinct clusters. Values of the polymorphic information content (PIC) value ranged from 0.0 to 0.66 with an average of 0.11 . Knowledge of the genetic diversity of castor can be used in future breeding programs for increased oil production to meet the ever increasing demand of castor oil for industrial uses as well as for biodiesel production.

\section{Introduction}

Castor (Ricinus communis L.) belongs to the family Euphorbiaceae. It has chromosome number $2 \mathrm{n}=20$. It is native to the tropical and sub-tropical regions continues to be an important non edible oil seed crop of arid and semi-arid regions of the world, for its high utilitarian value oil (Govaerts et al., 2000). India ranks 1st in terms of production of Castor oil seed in the world. Despite its name, castor is not a true bean, as it contains more than $45 \%$ oil and the oil is rich (80-90\%) in an unusual hydroxyl fatty acid, ricinoleic acid (Jeong and Park, 2009). Castor oil is used as raw material for numerous and varied 
industrial applications, such as: manufacture of polymers, coatings, lubricants for aircrafts, cosmetics etc. and also for the production of biofuel. Due to this increasing demand in the global market, there is a short supply of castor oil and this trend seems to get worst every year. Hence, new varieties need to be developed with high hectarage and productivity (Lakhani et al., 2015).

Castor is indigenous to Eastern Africa and most probably originated in Ethiopia. Castor is a highly polymorphic species; normally monoecious with pistillate flowers are situated on the upper part and staminate flowers on the lower part of raceme. Production of female and male flowers is highly influenced by environmental conditions. Though it is a cross-pollinated crop, most of the cultivars have been developed through hybridization followed by selection, as hybrids give significantly greater yields than pure lines or varieties (Moll et al., 1962; Birchler et al., 2003). Castor has a high degree of variation and offers the breeder ample scope to undertake screening and selection of seed sources for the desired traits (Reif et al., 2007). The previous studies paid more attention on morphological and agronomic trait variations and traits identification, e.g. diseases resistance, stress resistance. Knowledge of the genetic diversity is a fundamental aspect in the improvement of a crop species.

Selection is the most important activity in all plant breeding programmes (Zobel et al., 1984). Since, variability is a prerequisite for selection programmes, new genetic approaches like molecular marker technologies have been adopted to map genomes, in order to assess better cross combinations in developing better hybrids.

Genetic markers have polymorphic genetic properties which can be used to distinguish the parental origin of alleles (Andersen and Lubberstedt, 2003). Assessment of genetic variation using molecular markers is crucial for the efficient management and biodiversity conservation of plant genetic resources in gene banks. A large number of polymorphic markers are required to determine genetic relationships and genetic diversity in a reliable manner (Santalla et al., 1998). This limits the use of morphological and biochemical characters, which are limited in number or lack ample diversity in castor. Further, these analyses have intrinsic disadvantages such as limited numbers of markers, and are often less efficient due to their variation and sensitivity to short-term environmental fluctuations (Lesica et al., 1998). DNA based molecular analysis tools are ideal for germplasm characterization and phylogenetic studies (Masjoc, 2002).

Simple sequence repeats (SSR) or microsatellite marker on other hand require less amount of DNA sample without involvement of radioactive labels and is simpler as well as faster. Also SSR markers have ability to discriminate genotypes into homozygotes and heterozygotes due to the codominant nature. The objective of the present study was to investigated and characterized the genetic diversity present in the Indian genotypes of castor using SSR markers with an aim of accurate assessment of genetic diversity and select better cross combinations to develop popular hybrids with higher production potentials.

The information gathered here would be helpful in genomic mapping studies and for the development of castor genotypes with wider and diverse genetic background to obtained improved crop productivity. The identified polymorphic markers could also be exploited for improvement of castor through MAS and breeding as well as in future germplasm conservation strategies. 


\section{Materials and Methods}

\section{Collection and assemblage of plant materials}

The experimental material for the present study consisted of twenty castor genotypes collected from different parts of Gujarat. The list of species and their collection sites are furnished in Table 1.

\section{DNA isolation}

Seeds of each castor genotype were sown in pots and young leaves of 2 weeks old plants were collected from each genotype for DNA isolation. Genomic DNA was extracted from freshly harvested leaves of each castor genotype by adopting the procedure outlined by Purohit et al., (2013) with necessary modification.

\section{Quantification and electrophoresis of genomic DNA}

Agarose of $0.8 \%$ was melted and the gel was casted using gel casting plate. DNA samples were loaded in each well along with $5 \mu$ of loading dye and run at a constant voltage of $100 \mathrm{~V}$. The gel was placed over an UV-transilluminator and viewed at $300 \mathrm{~nm}$. The nucleic acid appeared as orange coloured intact band owing to the fluorescence of ethidium bromide and the gel was photographed using Alpha Innotech multiimage Digital 120 Zoom digital camera. The quantity and quality of the isolated DNA was determined by using PicoDrop N.D.1000 (Software Ver.3.3.2, Thermo Scientific, USA). Dilutions of 25 $\mathrm{ng} / \mu \mathrm{l}$ of each genotype were prepared and stored at $4{ }^{\circ} \mathrm{C}$ for further use in PCR analysis.

\section{SSR analysis}

Total thirty SSR primers were used for PCR amplification. Out of thirty SSR primers twenty-five were amplified and were used for further analysis. The PCR reactions were performed as per Galova et al., (2015) with some modifications. The PCR mixture comprised of $50 \mathrm{ng}$ genomic DNA, $10 \mathrm{mM}$ Tris- $\mathrm{HCl}$ ( $\mathrm{pH} 8.0$ ), $2.5 \mathrm{mM} \mathrm{MgCl} 2,1 \mathrm{U}$ Tag polymerase, 10 pmole primer and $200 \mu \mathrm{M}$ of dNTPs mixture (Bangalore Genei, India). The volume was made $15 \mu \mathrm{l}$ with sterile distilled water. The temperature cycle profiles were: an initial denaturation step for $5 \mathrm{~min}$ at $94^{\circ} \mathrm{C}$, followed by 35 cycles of denaturation step at $94^{\circ} \mathrm{C}$ for $1 \mathrm{~min}$, annealing at $57-60^{\circ} \mathrm{C}$ for 45 sec and primer extension at $72^{\circ} \mathrm{C}$ for $45 \mathrm{sec}$; final extension cycle of $7 \mathrm{~min}$ at $72^{\circ} \mathrm{C}$ was performed on Veriti 96-well Thermal Cycler.

All the above PCR amplification was performed in $0.2 \mathrm{ml}$ thin-walled PCR tubes placed in a thermal cycler (Veriti ${ }^{\circledR}$, Applied Biosystems). The products were analysed by electrophoresis in $1.5 \%$ agarose gel stained in ethidium bromide $(10 \mathrm{mg} / \mathrm{ml})$ and run in $1 \mathrm{x}$ TBE buffer at $100 \mathrm{~V}$ for $2 \mathrm{~h}$. The separated bands were visualized under UV transilluminator and photographed using a gel documentation system (BioRad). The amplified fragments were scored as 1 for the presence and 0 for the absence of a band from higher to lower molecular weight products. Faint bands were not recorded for analysis. By comparing the banding patterns of genotypes for a specific primer, genotype-specific bands were identified.

\section{Statistical analysis}

The molecular size of each fragment was estimated using Alpha Ease FC software (Alpha Innotech Corporation). SSR markers were scored as present (1) or absent (0) of a band, and the data obtained were used in a rectangular matrix. The data matrix was then used to generate a genetic similarity index (Nei and Li, 1979) using NTSYS 2.1 (Rohlf, 2000). The polymorphism information content 
(PIC) was calculated by the formula: PIC = 2Pi (1-Pi) (Bhat, 2002) where, Pi is the frequency of occurrence of polymorphic bands in different primers. Pairwise similarity matrices were generated by Jaccard's coefficient of similarity (Jaccard, 1908) by using the SIMQUAL format of NTSYSpc 2.1(Rohlf, 2000). Correlation between the two matrices obtained with two-marker types was estimated by means of the Mantel matrix correspondence test (Mantel, 1967). This test yields a product moment correlation $(r)$ that is one measure of the relatedness between the two matrices. A dendrogram was constructed by using the unweighted pair group method with arithmetic average (UPGMA) with the SAHN module of NTSYS-pc to show a phonetic representation of genetic relationships as revealed by the similarity coefficient (Sneath and Sokal, 1973).

\section{Results and Discussion}

Total plant genomic DNA was extracted from young leaves by Cetyl Trimethyl Ammonium Bromide (CTAB) method with some modifications. The absorbance ratio of DNA at A260/A280 ranged from 1.71 to 1.89 and the concentration ranged from 136.09 to $223.04 \mathrm{ng} / \mu 1$.

The twenty five primers showed polymorphism, out of the thirty used, producing a total of 36 bands/alleles, all of which were polymorphic representing $53.33 \%$ polymorphism with the number of amplified fragment varying with size ranging from 89 to $2190 \mathrm{bp}$. The number of amplified fragments produced per primer varying from 1 (Castor 6 , Castor 7, Castor 19, Castor 20, Castor 25, Castor 34, Castor 38, Castor 48, Castor 53, Castor 62, Castor 72, Castor 82, Castor 97, Castor 98, Castor 111, Castor 114, Castor 117 and Castor 123) to 5 (Castor 73) with average of 0.84 bands per primer (Table 1). The PIC values varied between 0.0 (Castor 6, Castor 7,
Castor 19, Castor 20, Castor 25, Castor 34, Castor 38, Castor 48, Castor 53, Castor 62, Castor72, Castor 82, Castor 97, Castor 98, Castor 111, Castor 114, Castor 117 and Castor 123) and 0.66 (Castor 8) with an average of 0.11 per primer (Table 1 ).

The dendrogram constructed by UPGMA method generated two main clusters that consists all the varieties grouped together in their respective sub-cluster. The SSR profile was visualized on $1.5 \%$ agarose gel and photographed. Only those fragments consistently amplified were scored for analysis. Similar to present finding, Maurya and Yadav (2016) studied the level of heterozygosity in Jatropha curcas using SSR markers. Out of 56 SSR primers, 18 primers were amplified. The polymorphic SSRs showed allele variation from 2 to 9 with an average of 3.56 alleles per primer. Earlier, Galova et al., (2015) analyzed 60 genotypes of castor using 10 SSR primers of Rco series and amplified 67 alleles ranging from 4 (Rco15) to 9 (Rco05) alleles with a mean value of 6.70 alleles per locus. Earlier, Costa et al., (2013) analyzed 66 sweet cassava accessions with 13 SSR primers of GA and SSRY series which amplified a total of 33 alleles with an average of 2.53 alleles per marker. Sakure et al., (2012) studied 22 castor genotypes using 20 SSR primers of SSRY series, out of which 10 SSR primers amplified, a total number of 353 fragments, out of which 309 were polymorphic, with an average of 9.8 polymorphic bands per primer.

The dendrogram obtain from UPGMA analysis grouped twenty genotypes into two main clusters with Jaccard similarity coefficient ranging from 0.62 to 1.00 (Table 2). Cluster one further divided into two subcluster-A and B both contained a total of 18 genotypes (Fig. 1). Subcluster-A was further bifurcated into two groups A1 and A2 having nearly $79 \%$ likeness. 
Table.1 Size, number of amplified bands, per cent polymorphism and PIC obtained by SSR primers in the 20 castor genotypes

\begin{tabular}{|c|c|c|c|c|c|c|c|c|c|c|}
\hline \multirow[t]{2}{*}{$\begin{array}{l}\text { Sr. } \\
\text { No. }\end{array}$} & \multirow[t]{2}{*}{$\begin{array}{c}\text { SSR } \\
\text { Primer }\end{array}$} & \multirow{2}{*}{$\begin{array}{l}\text { Band } \\
\text { Size } \\
\text { (bp) }\end{array}$} & \multirow{2}{*}{$\begin{array}{l}\text { Total No. } \\
\text { of Bands } \\
\text { (A) }\end{array}$} & \multicolumn{3}{|c|}{$\begin{array}{l}\text { Polymorphic } \\
\text { Bands (B) }\end{array}$} & \multirow{2}{*}{$\begin{array}{l}\text { Mono- } \\
\text { Morphic } \\
\text { Band }\end{array}$} & \multirow{2}{*}{$\begin{array}{l}\text { \% Poly- } \\
\text { Morphism } \\
\text { (B/A) }\end{array}$} & \multirow[t]{2}{*}{ PIC* } & \multirow[t]{2}{*}{ SPI } \\
\hline & & & & $\mathbf{S}$ & $\mathbf{U}$ & $\mathbf{T}$ & & & & \\
\hline 1 & Castor 6 & 139 & 1 & 0 & 0 & 0 & 1 & 0.0 & 0.0 & 0.0 \\
\hline 2 & Castor 7 & 228 & 1 & 0 & 0 & 0 & 1 & 0.0 & 0.0 & 0.0 \\
\hline 3 & Castor 8 & $175-432$ & 3 & 1 & 0 & 1 & 2 & 33.33 & 0.66 & 1.98 \\
\hline 4 & Castor 19 & 222 & 1 & 0 & 0 & 0 & 1 & 0.0 & 0.0 & 0.0 \\
\hline 5 & Castor 20 & 233 & 1 & 1 & 0 & 1 & 0 & 100.0 & 0.0 & 0.0 \\
\hline 6 & Castor 25 & 212 & 1 & 0 & 0 & 0 & 1 & 0.0 & 0.0 & 0.0 \\
\hline 7 & Castor 26 & $89-240$ & 2 & 0 & 1 & 1 & 1 & 50.0 & 0.09 & 0.18 \\
\hline 8 & Castor 34 & 236 & 1 & 1 & 0 & 1 & 0 & 100.0 & 0.0 & 0.0 \\
\hline 9 & Castor 38 & 153 & 1 & 1 & 0 & 1 & 0 & 100.0 & 0.0 & 0.0 \\
\hline 10 & Castor 42 & $254-306$ & 2 & 2 & 0 & 2 & 0 & 100.0 & 0.49 & 0.98 \\
\hline 11 & Castor 45 & $173-243$ & 2 & 2 & 0 & 2 & 0 & 100.0 & 0.50 & 1.0 \\
\hline 12 & Castor 48 & 205 & 1 & 1 & 0 & 1 & 0 & 100.0 & 0.0 & 0.0 \\
\hline 13 & Castor 53 & 252 & 1 & 0 & 0 & 0 & 1 & 0.0 & 0.0 & 0.0 \\
\hline 14 & Castor 62 & 237 & 1 & 0 & 0 & 0 & 1 & 0.0 & 0.0 & 0.0 \\
\hline 15 & Castor 72 & 245 & 1 & 1 & 0 & 1 & 0 & 100.0 & 0.0 & 0.0 \\
\hline 16 & Castor 73 & $191-2190$ & 5 & 2 & 3 & 5 & 0 & 100.0 & 0.53 & 2.65 \\
\hline 17 & Castor 78 & $93-183$ & 2 & 1 & 0 & 1 & 1 & 50.0 & 0.16 & 0.32 \\
\hline 18 & Castor 79 & $175-256$ & 2 & 1 & 0 & 1 & 1 & 50.0 & 0.49 & 0.98 \\
\hline 19 & Castor 82 & 222 & 1 & 0 & 0 & 0 & 1 & 0.0 & 0.0 & 0.0 \\
\hline 20 & Castor 97 & 192 & 1 & 1 & 0 & 1 & 0 & 100.0 & 0.0 & 0.0 \\
\hline 21 & Castor 98 & 135 & 1 & 0 & 0 & 0 & 1 & 0.0 & 0.0 & 0.0 \\
\hline 22 & Castor 111 & 231 & 1 & 1 & 0 & 1 & 0 & 100.0 & 0.0 & 0.0 \\
\hline 23 & Castor 114 & 214 & 1 & 1 & 0 & 1 & 0 & 100.0 & 0.0 & 0.0 \\
\hline 24 & Castor 117 & 206 & 1 & 1 & 0 & 1 & 0 & 100.0 & 0.0 & 0.0 \\
\hline 25 & Castor 123 & 208 & 1 & 0 & 0 & 0 & 1 & 0.0 & 0.0 & 0.0 \\
\hline \multicolumn{3}{|c|}{ TOTAL } & 36 & 18 & 4 & 22 & 14 & - & - & - \\
\hline \multicolumn{3}{|c|}{ AVERAGE } & - & - & - & 0.84 & 0.56 & 53.33 & 0.11 & 0.32 \\
\hline
\end{tabular}

$\mathrm{S}=$ Shared; $\mathrm{U}=$ Unique; $\mathrm{T}=$ Total polymorphic bands; $\mathrm{PIC}=$ Polymorphism information content; SPI = SSR primer index $=$ Number of bands $x$ PIC 
Table.2 Jaccard's similarity coefficient of 20 castor genotypes based on SSR

\begin{tabular}{|c|c|c|c|c|c|c|c|c|c|c|c|c|c|c|c|c|c|c|c|c|}
\hline & 1 & 2 & 3 & 4 & 5 & 6 & 7 & 8 & 9 & 10 & 11 & 12 & 13 & 14 & 15 & 16 & 17 & 18 & 19 & 20 \\
\hline 1 & 1.00 & & & & & & & & & & & & & & & & & & & \\
\hline 2 & 0.73 & 1.00 & & & & & & & & & & & & & & & & & & \\
\hline 3 & 0.87 & 0.80 & 1.00 & & & & & & & & & & & & & & & & & \\
\hline 4 & 0.84 & 0.66 & 0.84 & 1.00 & & & & & & & & & & & & & & & & \\
\hline 5 & 0.79 & 0.78 & 0.80 & 0.71 & 1.00 & & & & & & & & & & & & & & & \\
\hline 6 & 0.83 & 0.76 & 0.84 & 0.87 & 0.76 & 1.00 & & & & & & & & & & & & & & \\
\hline 7 & 0.74 & 0.67 & 0.75 & 0.77 & 0.72 & 0.89 & 1.00 & & & & & & & & & & & & & \\
\hline 8 & 0.90 & 0.71 & 0.84 & 0.81 & 0.77 & 0.81 & 0.72 & 1.00 & & & & & & & & & & & & \\
\hline 9 & 0.86 & 0.66 & 0.74 & 0.83 & 0.71 & 0.89 & 0.85 & 0.77 & 1.00 & & & & & & & & & & & \\
\hline 10 & 1.00 & 0.73 & 0.87 & 0.84 & 0.79 & 0.83 & 0.74 & 0.90 & 0.86 & 1.00 & & & & & & & & & & \\
\hline 11 & 0.81 & 0.73 & 0.76 & 0.68 & 0.68 & 0.77 & 0.69 & 0.78 & 0.73 & 0.81 & 1.00 & & & & & & & & & \\
\hline 12 & 0.77 & 0.70 & 0.84 & 0.87 & 0.70 & 0.93 & 0.89 & 0.75 & 0.89 & 0.77 & 0.72 & 1.00 & & & & & & & & \\
\hline 13 & 0.93 & 0.80 & 0.94 & 0.84 & 0.80 & 0.90 & 0.81 & 0.90 & 0.80 & 0.93 & 0.81 & 0.84 & 1.00 & & & & & & & \\
\hline 14 & 0.83 & 0.69 & 0.77 & 0.80 & 0.69 & 0.93 & 0.82 & 0.80 & 0.88 & 0.83 & 0.83 & 0.86 & 0.83 & 1.00 & & & & & & \\
\hline 15 & 0.77 & 0.69 & 0.77 & 0.80 & 0.69 & 0.93 & 0.89 & 0.80 & 0.88 & 0.77 & 0.77 & 0.93 & 0.83 & 0.92 & 1.00 & & & & & \\
\hline 16 & 0.73 & 0.66 & 0.74 & 0.77 & 0.71 & 0.89 & 0.92 & 0.71 & 0.85 & 0.73 & 0.68 & 0.89 & 0.80 & 0.81 & 0.88 & 1.00 & & & & \\
\hline 17 & 0.80 & 0.67 & 0.75 & 0.77 & 0.72 & 0.89 & 0.86 & 0.77 & 0.85 & 0.80 & 0.74 & 0.83 & 0.81 & 0.89 & 0.82 & 0.85 & 1.00 & & & \\
\hline 18 & 0.97 & 0.77 & 0.90 & 0.81 & 0.77 & 0.87 & 0.77 & 0.87 & 0.83 & 0.97 & 0.84 & 0.81 & 0.97 & 0.86 & 0.80 & 0.77 & 0.83 & 1.00 & & \\
\hline 19 & 1.00 & 0.73 & 0.87 & 0.84 & 0.79 & 0.83 & 0.74 & 0.90 & 0.86 & 1.00 & 0.81 & 0.77 & 0.93 & 0.83 & 0.77 & 0.73 & 0.80 & 0.97 & 1.00 & \\
\hline 20 & 0.76 & 0.62 & 0.71 & 0.73 & 0.74 & 0.85 & 0.81 & 0.68 & 0.81 & 0.76 & 0.70 & 0.79 & 0.77 & 0.85 & 0.78 & 0.81 & 0.88 & 0.79 & 0.76 & 1.00 \\
\hline
\end{tabular}


Fig.1 Dendrogram depicting the genetic relationship among 20 castor genotypes based on SSR markers.

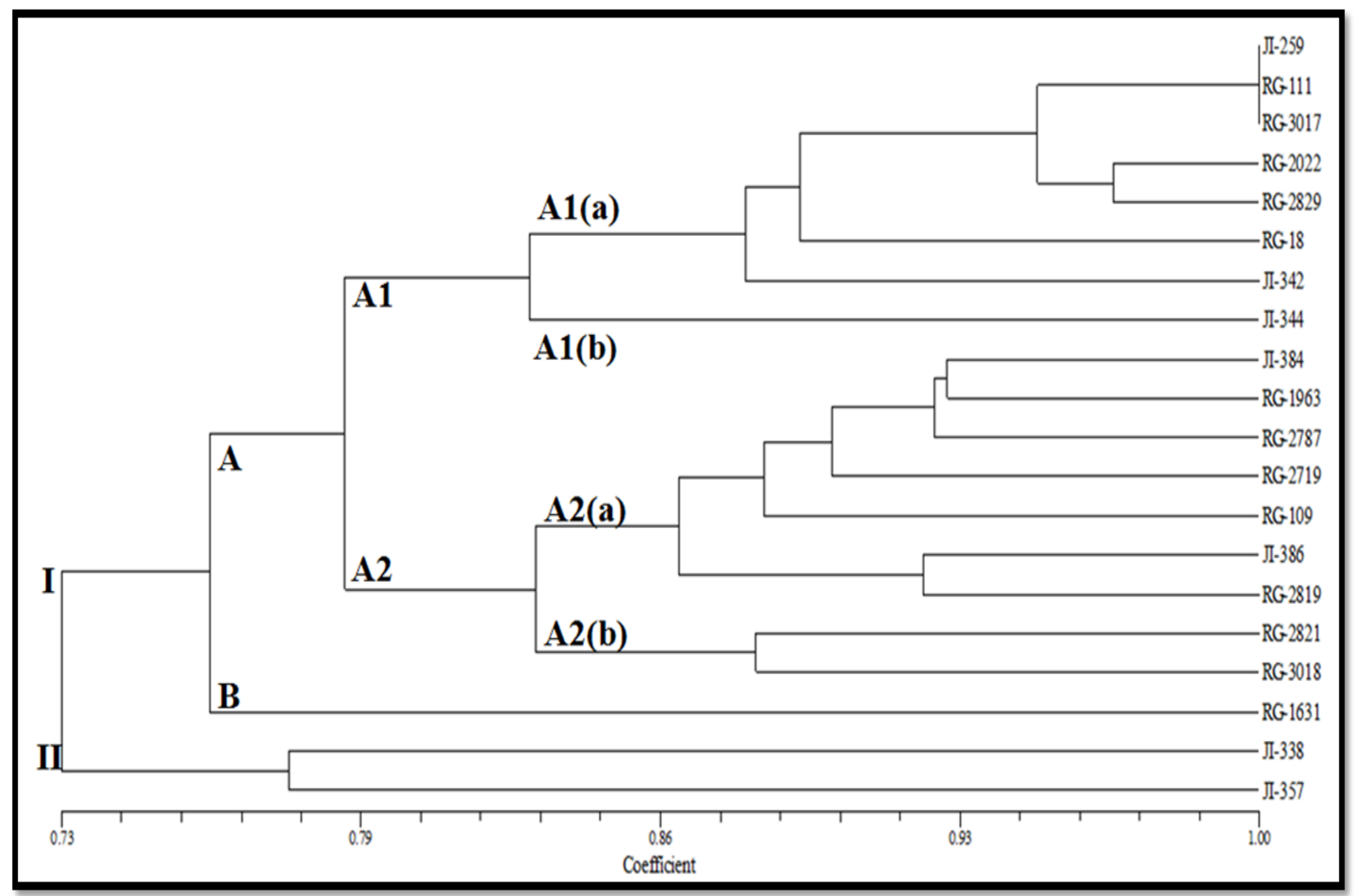


Group A1 was further divided into subgroup A1(a) and A1(b). Subgroup A1(a) consist of 7 genotypes JI-259, RG-111, RG-3017, RG2022, RG-2829, RG-18 having nearly $88 \%$ similarity while subgroup A1(b) consist of only one genotype JI-342. Group A2 was further divided into subgroup A2(a) and A2(b). Subgroup A2(a) consist of 7 genotypes JI-384，RG-1963，RG-2787，RG-2719，RG109. JI-386 and RG-2819 having nearly $87 \%$ similarity while subgroup A2(b) consist of two genotypes RG-2821 and RG-3018 having $88 \%$ similarity. Subcluster B consists of only one genotype RG-1631.

The technique used in the present investigation for analyzing the genetic diversity classified the twenty castor genotypes into distinct clusters based on the dendrogram prepared by UPGMA algorithm. Our analysis proved the utility of SSR markers for distinguishing the used set of castor genotypes. In conclusion, the present study indicated the prevalence of ample DNA polymorphism in selected castor genotypes which could be further utilized for breeding programs. The information gathered here would be helpful in genomic mapping studies with wider and diverse genetic background to obtained improved crop productivity.

\section{References}

Andersen, J.R., Lubberstedt, T. (2003). Functional marker in plants. Trends Plant Sci., 8(11): 554-560.

Bhat, K.V. (2002). Molecular data analysis. In: Proceedings of Short-Term Training Course on Molecular Maker Application in Plant Breeding, September 26-October 5, ICAR, NEW DELHI (India).

Birchler, J.A., Auger, D.L., Riddle, N.C. (2003). In search of the molecular basis of heterosis. Plant Cell 15, 2236-2239. CA, USA.
Costa, T.R., Filho, P.S., Vidigal, M.C., Galvan, M.Z., Lacanallo, G.F., Silva, L.I., Kvitschal, M.V. (2013). Genetic diversity and population structure of sweet cassava using simple sequence repeat (SSR) molecular markers. African J. Biotechnol., 12(10): 10401048.

Galova, Z., Vivodik, M., Balazova, Z., Hlozakova, T.K. (2015). Identification and differentiation of Ricinus communis L. using SSR markers. Potravinarstvo, 9(1): 556-561.

Govaerts, R., Frodin, D.G., Radcliffe-Smith, A. (2000). World Checklist and Bibliography of Euphorbiaceae (with Pandaceae). Redwood Books Limited, Trowbridge, Wiltshire.

Jaccard, P. (1908). Nouvelles recherches sur la distribution florale. Bull. Soc. Vaud. Sci. Nat., 44: 223-270.

Jeong, G.T., Park, D.H. (2009). Optimization of biodiesel production from castor oil using response surface methodology. Appl. Biochem. Biotechnol., 156: 431441.

Lakhani, H.N., Patel, S.V., Bodar, N.P., Golakiya, B.A. (2015). RAPD analysis of genetic diversity of castor bean (Ricinus communis L.). Inter. J. Curr. Microbiol. Appli. Sci., 4(1): 696-703.

Lesica, P., Leary, R.F., Allendort, F.R., Bilderbecl, D.E. (1998). Lack of genetic diversity within and among populations of an endangered plant, Hawellia aquatilis. Conserv Biol., 2: 275282.

Mantel, N. (1967). The detection of disease clustering and generalized regression approach. Cancer Res., 27: 209-220.

Masjoc, P. (2002). The application of molecular marker in process of selection. Cellular Mol. Biol. Letter, 7: 499-509.

Maurya, R., Yadav, H.K. (2016). Microsatellite markers based heterozygosity assessment in Jatropha 
curcas L.: A potential bioenergy crop. Tropical Pl. Res., 3(1): 191-198.

Moll, R.H., Salhuanaan, W.S., Robinson, D.H.F. (1962). Heterosis and genetic diversity in variety crosses of maize. Crop. Sci., 2: 197-198.

Nei, N., Li, W. (1979). Mathematical model for studying genetic variation in terms of restriction endonucleases. Proc Natl Acad Sci., USA, 76: 52695273.

Purohit, A.R., Verma, P.U., Patel, N.J. (2012). Rapid and efficient procedure for isolation of high yielding DNA from castor (Ricinus communis L.). Inter. J. Sci. Res. Pub., 2(7): 1-4.

Reif, J.C., Gumpert, F.M., Fischer, S., Melchinger, A.E. (2007). Impact of interpopulation divergence on additive and dominance variance in hybrid populations. Genetics, 176: 1931-1934.
Rohlf, F.J. (2000). NT-SYS-pc: Numerical Taxonomy and Multivariate Analysis System, Version 2.0 W. Exteer Software, Setauket.

Sakure, A.A., Dhaduk, H.L., Mehta, D.R., Madaria, R.B. (2012). Discrimination of castor (Ricinus communis L.) genotypes through SSR marker. Asian J. Biosci., 7(1): 71-76.

Santalla, M., Power, J.B., Davey, M.R. (1998). Genetic diversity in mungbean germplasm revealed by RAPD markers. Plant Breed, 117: 473-478.

Sneath, P.H.A., Sokal, R.R. (1973). Numerical Taxonomy. Freeman Press, San Francisco, CA, USA.

Zobel, B.J., Talbert, J.T. (1984). Applied forest tree improvement. John Wiley and Sons, New York, USA.

\section{How to cite this article:}

Harshita J. Kachhadiya, R.B. Madaria, Rushita V. Bhadani, Abhijeeta K. Nandha, Nidhi Savaliya and Virali Antala. 2019. Assessment of Molecular Diversity in Castor Genotypes Using SSR Markers. Int.J.Curr.Microbiol.App.Sci. 8(07): 595-603. doi: https://doi.org/10.20546/ijcmas.2019.807.074 